\title{
Prevalencia e influencia de la violencia homofóbica sobre la sintomatología depresiva y el nivel de autoestima.
}

\section{Prevalence and influence of homophobic violence on depressive symptomatology and the level of self- esteem.}

Fecha de recepción: 24-07-2020

Fecha de aceptación: 23-11-2020
Vicente Morell-Mengual Departamento de Psicología Evolutiva y de la Educación.

Universitat de València. España.

M. Dolores Gil-Llario Departamento de Psicología Evolutiva y de la Educación. Universitat de València. España.

Beatriz Gil-Juliá Departamento de Personalidad, Evaluación y Tratamientos Psicológicos.

Universitat de València. España.

\section{resumen/ahstract:}

Las personas homosexuales y bisexuales constituyen un colectivo sobre el que se ejerce violencia. Este estudio analiza la prevalencia e influencia que ejerce la violencia homofóbica sufrida a lo largo de la vida sobre la sintomatología depresiva y el nivel de autoestima. La muestra está compuesta por 730 personas homosexuales o bisexuales con edades comprendidas entre los 18 y los 60 años ( $M=28.47$; DT = 8.81). Los resultados indican que entre el $29.6 \%$ y $76.7 \%$ ha recibido violencia psicológica o verbal y entre el $4.2 \%$ y el $19.6 \%$ ha sufrido violencia física a lo largo de su vida. También se obtienen diferencias significativas según el género, siendo los hombres quienes refieren mayores tasas de victimización. Los análisis de regresión indican que la victimización en forma de risas, burlas, golpes y empujones disminuye el nivel de autoestima e incrementa la sintomatología depresiva. Estos resultados se discuten en relación a la necesidad de implementar programas educativos que prevengan este tipo de violencia o, al menos, reduzca su reiteración durante periodos prolongados.

Gay and bisexual people constitute a group on which violence is exercised. This study analyses the prevalence and influence exerted by homophobic violence suffered throughout life on depressive symptoms and the level of selfesteem. The sample is made up of 730 homosexual or bisexual people with ages between 18 and 60 years $(M=28.47$; $S D=8.81)$. The results indicate that between $29.6 \%$ and $76.7 \%$ have received psychological or verbal violence and between $4.2 \%$ and $19.6 \%$ have suffered physical violence throughout their lives. Significant differences according to gender are also obtained, with men reporting higher victimization rates. Regression analysis show that victimization as laughter, teasing, hitting and shoving decreases the level of self-esteem and increases depressive symptoms. These results are discussed in relation to the need of implementing educational programs that prevent this type of violence or, at least, reduce its repetition for prolonged periods.

\section{palabras clave/keywords:}

bullying homofóbico; violencia homofóbica; depresión; autoestima.

homophobic bullying; homophobic violence; depression; self esteem. 


\section{Introducción}

En la actualidad España constituye uno de los países con una actitud más positiva hacia la diversidad afectivo-sexual, así como con menores tasas de violencia homofóbica, sólo por detrás de países nórdicos como Noruega o Suecia (Petrou y Lemke 2018). Si bien los cambios sociales y de legislación acontecidos en las últimas décadas parecen haber favorecido un clima de mayor tolerancia y respeto hacia la diversidad afectivo-sexual en las sociedades occidentales (Worthen, Lingiardi y Caristo, 2017), la victimización por motivos de orientación sexual sigue siendo una realidad basada en la heteronormatividad y los roles de género que prevalecen en nuestra cultura (Carrera-Fernández, Cid-Fernández, Almeida, GonzálezFernández y Lameiras-Fernández, 2018). De esta manera la heterosexualidad se impone como la sexualidad más aceptada, lo que conlleva a su vez la persecución y la marginación de las personas no heterosexuales (UNESCO, 2015). No obstante, esta violencia no sólo estaría dirigida a personas de una orientación sexual diferente a la norma culturalmente establecida, sino también a aquellas personas cuyo comportamiento no refleja los patrones de género socialmente establecidos (Platero, 2008). Esta violencia más bien se fundamenta en la orientación sexual percibida y no tanto en la orientación sexual referida pudiendo ser víctimas de violencia homofóbica también las personas que aun reconociéndose como heterosexuales son percibidas como gays, lesbianas o bisexuales (Poteat, Scheer, DiGiovanni y Mereish, 2014), así como también aquellas que apoyan a las víctimas de acoso o violencia por homofobia por el llamado "contagio del estigma" (Pichardo, de Stéfano, Faure, Sáenz y williamns, 2015).

La actitud hostil y discriminatoria a la que se ven sometidas las personas por motivos de orientación sexual puede expresarse de diferentes formas como señalan Pichardo, Molinuevo, Rodríguez, Martín y Romero (2007): formas activas de violencia física y/o verbal, rechazo silencioso e institucionalizado de las personas identificadas como homosexuales y la limitación a su acceso a derechos, espacios, reconocimiento, prestigio o poder. Además, estas situaciones se viven en prácticamente todos los ámbitos en los que se desenvuelve la persona, desde los más privados hasta los más públicos, siendo el contexto escolar uno en los que esta violencia es reproducida si cabe con mayor fuerza (Pichardo et al., 2007).

Los estudios de prevalencia muestran sistemáticamente cifras más altas de acoso entre estudiantes LGTBQ (Elipe, de la Oliva y Del Rey, 2018) con valores que oscilan entre el 57$70 \%$ (Baruch, 2012; Marchueta, 2014) e incluso llegando al 85\% cuando se hace referencia al acoso verbal (Kosciw, Greytak, Giga, Villenas, y Danischewski, 2016). En este sentido, un estudio llevado a cabo en nuestro contexto (FELGTB, 2012) con 653 adolescentes y jóvenes que han experimentado o experimentan acoso escolar por ser o parecer lesbianas, gays o bisexuales (LGB) revela que la violencia más frecuente es la verbal o psicológica a través de burlas e imitaciones sarcásticas (64\%), rumores sobre su persona (69\%), insultos (71\%) y comentarios despectivos por ser LGB (72\%). En segundo lugar, destaca la violencia social o relacional marcada por el aislamiento o exclusión $(37 \%)$ e incluso ignorarles por ser LGB (39\%). En tercer lugar, respecto a la violencia física, un 5\% ha recibido palizas, un $6 \%$ ha sido víctima de acoso o agresiones sexuales, un $23 \%$ ha sufrido amenazas y 
un 36\% ha recibido golpes o empujones. En esta misma línea, Blondeel y cols. (2018) en su revisión sistemática obtuvieron datos de prevalencia de violencia física y sexual en adultos de entre $6 \%-25 \%$ y $5.6 \%-11.4 \%$, respectivamente.

Cabe señalar que estas actitudes homófobas aparecen con mayor frecuencia entre los chicos (Pichardo et al., 2007), siendo a su vez los chicos gays y bisexuales quienes más frecuentemente resultan víctimas de violencia frente a las chicas lesbianas y bisexuales (Cereceda et al., 2018; D'haese, Dewaele y Van Houtte, 2016), en prácticamente todos los tipos de violencia, si bien en algunos estudios no se obtuvieron diferencias en función del género en la violencia verbal (D’haese et al., 2016).

Asimismo, desde este marco es importante tener en cuenta que la victimización por motivos de orientación sexual supone gran sufrimiento y consecuencias negativas sobre la salud mental de las personas afectadas (Albaladejo-Blázquez et al., 2019; D'haese et al., 2016; FELGTB, 2012). Diversos estudios revelan que estas experiencias discriminatorias y de hostigamiento están relacionadas con la presencia de síntomas de depresión (Marchueta, 2014; Teasdale y Bradley-Engen, 2010), baja autoestima (Huebner, Rebchook y Kegeles, 2004; Marchueta, 2014), consumo/abuso de sustancias (Lehavot y Simoni, 2011) e ideación y /o conducta suicida (Teasdale y Bradley-Engen, 2010). Por tanto, la violencia homofóbica en cualquiera de sus formas se asocia a peor salud mental, sin subestimar el impacto y severidad de la violencia homofóbica verbal, sobre todo teniendo en cuenta su alta prevalencia, cuyos efectos han mostrado ser similares al resto de formas de violencia (D'haese et al., 2016) y con efectos devastadores especialmente sobre la autoestima (Huebner et al., 2004).

Dada la relevancia del tema y la escasez de estudios llevados a cabo en nuestro contexto, el objetivo de este trabajo ha sido analizar la prevalencia y severidad de la violencia homofóbica, valorar posibles diferencias en función del género, así como su relación con la sintomatología depresiva y el nivel de autoestima.

\section{Método}

\section{Participantes}

La muestra está compuesta por 730 personas, 404 hombres (55.3\%) y 326 mujeres (44.7\%) con edades comprendidas entre los 18 y los 60 años $(\mathrm{M}=28.47$; DT $=8.81)$, procedentes de diversos puntos de la geografía española. Respecto a la orientación sexual, el $72.7 \%$ se identificó como homosexual (348 hombres y 183 hombres) y el $27.3 \%$ como bisexual (56 hombres y 143 mujeres). Por nivel educativo, el $40.8 \%$ poseía estudios secundarios y el $58.2 \%$ estudios universitarios, mientras que sólo el $1 \%$ poseía estudios primarios. Respecto a la distribución por nacionalidad, 91.2\% eran españoles y 7.9\% inmigrantes, predominando nacionalidades como la mexicana y la colombiana. La tabla 1 recoge todos los datos sociodemográficos para el conjunto de la muestra, así como para hombres y mujeres.

\section{Instrumentos}

GVH-7. Escala de gravedad de la violencia homofóbica (Ballester-Arnal, Morell-Mengual, Gil-Llario, Salmerón-Sánchez y Castro-Calvo, 2015). Instrumento compuesto por 7 ítems que evalúa la severidad de la victimización homofóbica en relación a tres tipos de violencia: 
Tabla 1.- Datos sociodemográficos

\begin{tabular}{lccc}
\hline & Hombres $(\mathbf{n}=\mathbf{4 0 4})$ & Mujeres $(\mathbf{n}=\mathbf{3 2 6})$ & Total $(\mathbf{n = 7 3 0})$ \\
\hline Orientación sexual & & & \\
Homosexual & $86.1 \%$ & $43.9 \%$ & $72.7 \%$ \\
Bisexual & $13.9 \%$ & $56.1 \%$ & $27.3 \%$ \\
Edad & & & \\
Menores de 20 & $9.9 \%$ & $8.6 \%$ & $9.3 \%$ \\
Entre 20 y 29 & $54 \%$ & $58.3 \%$ & $55.9 \%$ \\
Entre 30 y 39 & $21.3 \%$ & $23.9 \%$ & $22.5 \%$ \\
Entre 40 y 50 & $11.1 \%$ & $6.7 \%$ & $9.2 \%$ \\
Mayores de 50 & $3.7 \%$ & $2.5 \%$ & $3.2 \%$ \\
Nivel educativo & & & \\
Primarios & $0.7 \%$ & $1.2 \%$ & $40.8 \%$ \\
Secundarios & $39.2 \%$ & $42.9 \%$ & $58.2 \%$ \\
Universitarios & $60.1 \%$ & $55.9 \%$ & \\
Nacionalidad & & & $93 \%$ \\
Española & $92.1 \%$ & $94.2 \%$ & $7 \%$ \\
Otra & $7.9 \%$ & $5.8 \%$ & \\
\hline
\end{tabular}

psicológica, relacional y física. Se contesta mediante una escala tipo Likert de 5 puntos, donde debe indicarse la frecuencia con la que se experimentan los diversos comportamientos recogidos en cada ítem: $0=$ Nunca, $1=$ Raramente, $2=A$ veces, $3=$ A menudo o habitualmente, 4 = Muy a menudo o a diario. El índice de consistencia interna obtenida por los autores originales revela una buena fiabilidad $(\alpha=.83)$. El Alpha de Cronbach de la escala obtenido en este estudio es de .86 .

CESD-7. Escala de Depresión del Centro de Estudios Epidemiológicos (Herrero y Gracia, 2007; Radloff, 1977). Escala compuesta por 7 ítems (por ejemplo, «me costaba concentrarme en lo que estaba haciendo» o «me sentí deprimido») que evalúa algunos aspectos relacionados con la sintomatología depresiva. Se contesta indicando la frecuencia que mejor describe cómo se ha sentido la persona durante la última semana, según las siguientes alternativas: 1 = menos de un día; $2=$ uno o dos días; $3=$ tres o cuatro días y $4=$ cinco o siete días. El rango de puntuación oscila entre 7 y 28. La adaptación y validación española presenta un coeficiente de fiabilidad alfa de Cronbach de .82. En el presente estudio se obtiene un Alpha de Cronbach de .86 .

RSES. Escala de Autoestima de Rosenberg (Martín-Albo, Núñez, Navarro y Grijalvo, 2007; Rosenberg, 1965). Instrumento compuesto por 10 ítems que evalúa la autoestima personal entendida como los sentimientos de valía personal y de respeto a sí mismo (por ejemplo: «creo que no tengo mucho de lo que estar orgulloso» о «en general me siento satisfecho 
conmigo mismo»). Se responde con una escala tipo Likert de cuatro opciones que van desde 1 «totalmente en desacuerdo» a 4 «totalmente de acuerdo». El rango de puntuación oscila entre 10 y 40 . La consistencia interna de la adaptación y validación española, evaluada mediante el coeficiente alfa de Cronbach, revela una buena fiabilidad tanto en la primera administración $(\alpha=.85)$ como en la segunda $(\alpha=.88)$. El Alpha de Cronbach para la muestra del presente estudio es de .89 .

\section{Procedimiento}

La muestra fue reclutada de forma online. En primer lugar, se contactó con diversas asociaciones y colectivos pertenecientes a la Federación Española de Lesbianas, Gays, Transexuales (FELGTB), requiriéndoles su colaboración y facilitándoles información sobre el estudio a desarrollar. En cada territorio se contactó con un número suficiente de asociaciones para asegurar la representatividad y la extrapolación de los datos. A continuación, las asociaciones que finalmente aceptaron colaborar publicaron, a través de sus redes sociales (Facebook y Twitter, principalmente) o sus páginas webs, un mensaje que contenía una breve descripción del estudio junto a un enlace que remitía a la batería de cuestionarios. Cuando el participante accedía a la encuesta, se le proporcionaba una información más amplia sobre los objetivos del estudio y era necesaria la obtención de su consentimiento informado para proceder a la cumplimentación de la misma. En todo momento se garantizó el anonimato y la confidencialidad de los datos proporcionados. La participación fue voluntaria.

\section{Análisis de datos}

En primer lugar, se realizan análisis descriptivos para extraer la prevalencia de las distintas conductas violentas y la posible existencia de diferencias en función del género mediante una prueba chi cuadrado. A continuación, se establecieron tres grupos de contraste utilizando como criterio las respuestas de la escala de gravedad de la violencia homofóbica (Ballester-Arnal et al., 2015). Los sujetos que refirieron haber sufrido un determinado comportamiento «muy a menudo» $\mathrm{y}$ «a menudo» fueron asignados al grupo de alta severidad, mientras que aquellos que indicaron haber sido victimizados «a veces» $\mathrm{y}$ «raramente» fueron asignados al grupo de baja severidad. Los sujetos que nunca habían sido victimizados fueron asignados al grupo de no víctimas. Finalmente, se realizó un ANOVA para comprobar la posible existencia de diferencias en dos variables relacionadas con la salud mental, la sintomatología depresiva y el nivel de autoestima. Todos los análisis se realizaron con el paquete estadístico IBM SPSS Statistics 26.

\section{Resultados}

\section{Prevalencia y severidad de la violencia homofóbica}

Los datos obtenidos indican que la violencia verbal, relacional y física constituye una vivencia experimentada por un elevado porcentaje de hombres y mujeres homosexuales. Así, los comportamientos más reportados están circunscritos al ámbito de la violencia verbal como los insultos y las ridiculizaciones (76.7\%), hablar mal o divulgar rumores falsos (73.4\%) o las risas y las burlas (70.7\%). Contrariamente, las conductas que implican violencia física, como golpes o empujones y palizas presentan menor prevalencia, $19.6 \%$ y $4.2 \%$, respectivamente. 
En general, la violencia homofóbica no suele prolongarse en el tiempo. Un elevado porcentaje de la muestra refiere haber sufrido este tipo de violencia raramente o a veces, con porcentajes varían entre el $83 \%$ y el $90 \%$ en función del tipo de victimización. No obstante, cabe destacar que el $9.7 \%$ refiere haber sufrido palizas muy a menudo, constituyendo la conducta que mayor riesgo entraña para la integridad física.

\section{Prevalencia y severidad de la violencia homofóbica: diferencias de género}

Analizando las diferencias que surgen en función del género (ver tabla 2), los hombres presentan mayores índices de violencia homofóbica en todos los comportamientos analizados con respecto a las mujeres, si bien, las diferencias sólo son estadísticamente significativas en los insultos o ridiculizaciones $(81.1 \%$ vs. $70.2 \% ; \mathrm{p}=.001)$, las risas o burlas $(79 \%$ vs. $60.4 \% ; \mathrm{p}=.001$ ) y los golpes o empujones ( $24 \%$ vs. $14.1 \% ; \mathrm{p}=.001$ ). No obstante, también es importante comparar las diferencias respecto a la frecuencia con la que se han sufrido dichos comportamientos. En general, las mujeres refieren mayor reiteración de actitudes y comportamientos que entrañan violencia verbal y violencia física de carácter leve. Considerando las respuestas que entrañan mayor gravedad («a menudo» y «muy a menudo»), el $21 \%$ ha experimentado la divulgación de rumores falsos, el $16.3 \%$ ha sido víctima de amenazas y el $13 \%$ ha recibido golpes o empujones. Por el contrario, los hombres presentan mayor severidad en comportamientos que suponen niveles de violencia física muy grave. Así, el 22.3\% refiere haber recibido palizas muy a menudo o a menudo en contraposición a las mujeres quienes únicamente reseñan haber sufrido esta victimización de forma poco prolongada en el tiempo. Finalmente, otros comportamientos relacionados con los insultos, las burlas o la exclusión social presentan porcentajes de severidad que no difieren significativamente entre hombres y mujeres.

\section{Depresión y autoestima según el nivel de intensidad de la violencia homofóbica}

El análisis de varianza revela la existencia de diferencias estadísticamente significativas entre los grupos de severidad baja y alta en comparación con quienes no han sufrido violencia homofóbica en las dos variables analizadas (ver tabla 3). En cuanto al nivel de autoestima, las personas que no han padecido violencia física en forma de golpes y/o empujones presentan mayores niveles de autoestima que quienes han sufrido esas conductas con baja ( $\mathrm{p}=$ .033) o alta intensidad ( $\mathrm{p}=.005)$, indistintamente. De igual modo, quienes han sido objeto de risas y burlas $(\mathrm{p}=.006)$ o amenazas severas $(\mathrm{p}=.009)$ poseen menor autoestima en comparación con quienes no han sufrido este tipo de violencia.

Las personas que han sido sufrido violencia verbal (insultos, divulgación de rumores falsos, burlas, risas y/o amenazas) y violencia física (golpes, empujones y/o palizas) en grado severo, presentan mayores niveles de sintomatología depresiva y menores niveles de autoestima que los demás grupos analizados, siendo estas diferencias estadísticamente significativas. En contraposición, el resto de conductas (recibir insultos, soportar ridiculizaciones sarcásticas o que se les retire la palabra) no revelan diferencias en cuanto a la sintomatología depresiva o a la autoestima entre los tres niveles de gravedad de victimización analizados. 
Tabla 2.- Prevalencia y frecuencia de la victimización homofóbica

\begin{tabular}{|c|c|c|c|c|c|c|c|c|}
\hline & & \multicolumn{3}{|c|}{ Victimización } & \multicolumn{2}{|c|}{ Frecuencia } & \multirow[b]{2}{*}{$\begin{array}{l}\text { Muy a } \\
\text { menudo }\end{array}$} & \multirow[b]{2}{*}{$\mathrm{X}^{2}$} \\
\hline & & $\mathrm{Si}$ & $\mathrm{X}^{2}$ & Raramente & A veces & $\begin{array}{c}\text { A } \\
\text { menudo }\end{array}$ & & \\
\hline \multirow{3}{*}{$\begin{array}{l}\text { Insultos o } \\
\text { ridiculizaciones }\end{array}$} & Hombres & $81.1 \%$ & \multirow{3}{*}{$13.790^{* * *}$} & $38.1 \%$ & $50.8 \%$ & $8.2 \%$ & $3 \%$ & \multirow{3}{*}{5.570} \\
\hline & Mujeres & $70.2 \%$ & & $46.7 \%$ & $43.7 \%$ & $8.3 \%$ & $1.3 \%$ & \\
\hline & Total & $76.7 \%$ & & $41.6 \%$ & $47.9 \%$ & $8.2 \%$ & $2.3 \%$ & \\
\hline \multirow{3}{*}{$\begin{array}{l}\text { Hablar mal } \\
\text { o divulgar } \\
\text { rumores falsos }\end{array}$} & Hombres & $69.9 \%$ & \multirow{3}{*}{3.669} & $38 \%$ & $48.7 \%$ & $10.4 \%$ & $2.9 \%$ & \multirow{3}{*}{$14.565^{* *}$} \\
\hline & Mujeres & $76.2 \%$ & & $34.6 \%$ & $44.3 \%$ & $20.6 \%$ & $0.4 \%$ & \\
\hline & Total & $73.4 \%$ & & $36.6 \%$ & $46.8 \%$ & $14.7 \%$ & $1.9 \%$ & \\
\hline \multirow{3}{*}{ Risas o burlas } & Hombres & $79 \%$ & \multirow{3}{*}{$29.899^{* * *}$} & $38.9 \%$ & $44.2 \%$ & $13.5 \%$ & $3.4 \%$ & \multirow{3}{*}{1.153} \\
\hline & Mujeres & $60.4 \%$ & & $43.1 \%$ & $42.1 \%$ & $12.2 \%$ & $2.5 \%$ & \\
\hline & Total & $70.7 \%$ & & $40.5 \%$ & $43.5 \%$ & $12.9 \%$ & $3.1 \%$ & \\
\hline \multirow{3}{*}{ Amenazas } & Hombres & $32.2 \%$ & \multirow{3}{*}{2.911} & $65.4 \%$ & $26.2 \%$ & $5.4 \%$ & $3.1 \%$ & \multirow{3}{*}{6.517} \\
\hline & Mujeres & $26.4 \%$ & & $50 \%$ & $33.7 \%$ & $12.8 \%$ & $3.5 \%$ & \\
\hline & Total & $29.6 \%$ & & $59.3 \%$ & $29.2 \%$ & $8.3 \%$ & $3.2 \%$ & \\
\hline \multirow{3}{*}{$\begin{array}{l}\text { Golpes o } \\
\text { empujones }\end{array}$} & Hombres & $24 \%$ & \multirow{3}{*}{$11.225^{* * *}$} & $60.8 \%$ & $32 \%$ & $3.1 \%$ & $4.1 \%$ & \multirow{3}{*}{6.968} \\
\hline & Mujeres & $14.1 \%$ & & $58.7 \%$ & $28.3 \%$ & $13 \%$ & $0 \%$ & \\
\hline & Total & $19.6 \%$ & & $60.1 \%$ & $30.8 \%$ & $6.3 \%$ & $2.8 \%$ & \\
\hline \multirow{3}{*}{ Palizas } & Hombres & $4.5 \%$ & \multirow{3}{*}{.097} & $61.1 \%$ & $16.7 \%$ & $5.6 \%$ & $16.7 \%$ & \multirow{3}{*}{4.279} \\
\hline & Mujeres & $4 \%$ & & $61.5 \%$ & $38.5 \%$ & $0 \%$ & $0 \%$ & \\
\hline & Total & $4.2 \%$ & & $61.3 \%$ & $25.8 \%$ & $3.2 \%$ & $9.7 \%$ & \\
\hline \multirow{3}{*}{ Dejar de hablar } & Hombres & $44.6 \%$ & \multirow{3}{*}{2.657} & $51.7 \%$ & $33.9 \%$ & $8.9 \%$ & $5.6 \%$ & \multirow{3}{*}{7.364} \\
\hline & Mujeres & $50.6 \%$ & & $43 \%$ & $44.8 \%$ & $10.3 \%$ & $1.8 \%$ & \\
\hline & Total & $47.3 \%$ & & $47.5 \%$ & $39.1 \%$ & $9.6 \%$ & $3.8 \%$ & \\
\hline
\end{tabular}

Nota: ${ }^{* *} \mathrm{p}>.01 ;{ }^{* * *} \mathrm{p}>.001$ 
Tabla 3.- Depresión y autoestima según el nivel de intensidad de la victimización homofóbica

\begin{tabular}{|c|c|c|c|c|c|c|c|}
\hline & & \multicolumn{3}{|c|}{ Depresión } & \multicolumn{3}{|c|}{ Autoestima } \\
\hline & & $\begin{array}{c}\text { M } \\
\text { (DT) }\end{array}$ & $\mathbf{F}$ & Bonferroni & $\begin{array}{c}\text { M } \\
\text { (DT) }\end{array}$ & $\mathbf{F}$ & Bonferroni \\
\hline \multirow{3}{*}{$\begin{array}{l}\text { Insultos o } \\
\text { ridiculizaciones }\end{array}$} & NV & $\begin{array}{l}12.49 \\
(4.54)\end{array}$ & \multirow{3}{*}{2.019} & & $\begin{array}{l}32.62 \\
(6.77)\end{array}$ & \multirow{3}{*}{2.156} & \multirow{6}{*}{$\begin{array}{c}\mathrm{NV}>\mathrm{SA}^{* *} \\
\mathrm{SB}>\mathrm{SA}^{*}\end{array}$} \\
\hline & SB & $\begin{array}{l}13.25 \\
(4.67)\end{array}$ & & & $\begin{array}{l}31.73 \\
(6.44)\end{array}$ & & \\
\hline & SA & $\begin{array}{l}13.59 \\
(5.05)\end{array}$ & & & $\begin{array}{l}30.73 \\
(6.18)\end{array}$ & & \\
\hline \multirow{3}{*}{$\begin{array}{l}\text { Hablar mal o } \\
\text { divulgar rumores } \\
\text { falsos }\end{array}$} & NV & $\begin{array}{l}12.61 \\
(4.47)\end{array}$ & \multirow{3}{*}{$8.934^{* * *}$} & & $\begin{array}{l}32.62 \\
(6.50)\end{array}$ & \multirow{3}{*}{$5.216^{* *}$} & \\
\hline & SB & $\begin{array}{l}12.94 \\
(4.58)\end{array}$ & & $\begin{array}{l}\mathrm{SA}>\mathrm{NV}^{* * * *} \\
\mathrm{SA}>\mathrm{SB}^{* * *}\end{array}$ & $\begin{array}{l}31.90 \\
(6.44)\end{array}$ & & \\
\hline & SA & $\begin{array}{l}15.01 \\
(5.19)\end{array}$ & & & $\begin{array}{l}29.96 \\
(6.56)\end{array}$ & & \\
\hline \multirow{3}{*}{ Risas o burlas } & NV & $\begin{array}{l}12.40 \\
(4.57)\end{array}$ & \multirow{3}{*}{$4.366^{* *}$} & & $\begin{array}{l}32.90 \\
(6.56)\end{array}$ & \multirow{3}{*}{$5.486^{* *}$} & \multirow{3}{*}{$\mathrm{NV}>\mathrm{SA}^{* *}$} \\
\hline & SB & $\begin{array}{l}13.27 \\
(4.60)\end{array}$ & & $\mathrm{SA}>\mathrm{NV}^{*}$ & $\begin{array}{l}31.64 \\
(6.40)\end{array}$ & & \\
\hline & SA & $\begin{array}{l}14.04 \\
(5.18)\end{array}$ & & & $\begin{array}{l}30.29 \\
(6.56)\end{array}$ & & \\
\hline \multirow{3}{*}{ Amenazas } & NV & $\begin{array}{l}12.86 \\
(4.58)\end{array}$ & \multirow{3}{*}{2.617} & & $\begin{array}{l}32.26 \\
(6.46)\end{array}$ & \multirow{3}{*}{$5.644^{* *}$} & \multirow{3}{*}{$\mathrm{NV}>\mathrm{SA}^{* *}$} \\
\hline & SB & $\begin{array}{l}13.58 \\
(4.74)\end{array}$ & & & $\begin{array}{l}31.23 \\
(6.45)\end{array}$ & & \\
\hline & SA & $\begin{array}{l}14.40 \\
(5.92)\end{array}$ & & & $\begin{array}{l}28.32 \\
(6.78)\end{array}$ & & \\
\hline \multirow{3}{*}{$\begin{array}{l}\text { Golpes o } \\
\text { empujones }\end{array}$} & NV & $\begin{array}{l}12.86 \\
(4.52)\end{array}$ & \multirow{3}{*}{$4.431^{*}$} & & $\begin{array}{l}32.21 \\
(6.41)\end{array}$ & \multirow{3}{*}{$5.701^{* *}$} & \multirow{9}{*}{$\begin{array}{l}\mathrm{NV}>\mathrm{SB}^{*} \\
\mathrm{NV}>\mathrm{SA}^{* *}\end{array}$} \\
\hline & SB & $\begin{array}{l}14.02 \\
(4.99)\end{array}$ & & $\begin{array}{l}\mathrm{SB}>\mathrm{NV}^{*} \\
\mathrm{SA}>\mathrm{NV}^{*}\end{array}$ & $\begin{array}{l}30.62 \\
(6.54)\end{array}$ & & \\
\hline & SA & $\begin{array}{l}14.99 \\
(7.07)\end{array}$ & & & $\begin{array}{l}27.92 \\
(8.22)\end{array}$ & & \\
\hline \multirow{3}{*}{ Palizas } & NV & $\begin{array}{l}13.01 \\
(4.59)\end{array}$ & \multirow{3}{*}{$4.009^{*}$} & & $\begin{array}{l}31.95 \\
(6.47)\end{array}$ & \multirow{3}{*}{1.763} & \\
\hline & SB & $\begin{array}{l}15.37 \\
(5.54)\end{array}$ & & $\begin{array}{l}\mathrm{SB}>\mathrm{NV}^{*} \\
\mathrm{SA}>\mathrm{NV}^{*}\end{array}$ & $\begin{array}{l}29.59 \\
(6.89)\end{array}$ & & \\
\hline & SA & $\begin{array}{c}15.75 \\
(10.11)\end{array}$ & & & $\begin{array}{l}30.75 \\
(9.78)\end{array}$ & & \\
\hline \multirow{3}{*}{ Dejar de hablar } & NV & $\begin{array}{l}12.80 \\
(4.40)\end{array}$ & \multirow{3}{*}{2.058} & & $\begin{array}{l}32.09 \\
(6.39)\end{array}$ & \multirow{3}{*}{.572} & \\
\hline & SB & $\begin{array}{l}13.36 \\
(4.85)\end{array}$ & & & $\begin{array}{l}31.62 \\
(6.63)\end{array}$ & & \\
\hline & SA & $\begin{array}{l}13.96 \\
(5.62)\end{array}$ & & & $\begin{array}{l}31.37 \\
(6.51)\end{array}$ & & \\
\hline
\end{tabular}

Nota: NV: no víctima; SB: severidad baja; SA; severidad alta.

Nota: ${ }^{*} \mathrm{p}>.05 ;{ }^{* *} \mathrm{p}>.01 ;{ }^{* * *} \mathrm{p}>.001$. 


\section{Discusión}

El objetivo de este trabajo ha sido analizar la prevalencia de la violencia homofóbica sufrida a lo largo de la vida y su relación con la sintomatología depresiva y el nivel de autoestima. Aunque existen numerosos trabajos que analizan las repercusiones del bullying homofóbico sobre la salud mental de los adolescentes (Elipe et al., 2018; Garaigordobil y Larrain, 2020), todavía no existe suficiente evidencia empírica que permita determinar si las consecuencias de este tipo de victimización se mantienen en la adultez.

Un elevado porcentaje de personas homosexuales o bisexuales ha sufrido violencia homofóbica debido a su orientación sexual (Kosciw et al., 2016). Sin embargo, la prevalencia varía según la conducta analizada, observándose mayores tasas de victimización en aquellas acciones circunscritas al ámbito de la violencia psicológica y verbal frente a situaciones relacionadas con la intimidación y la violencia física. Estos datos son congruentes con diversos estudios que indican mayores tasas de violencia verbal en forma de insultos, divulgación de rumores o burlas (Marchueta, 2014; Martxueta y Etxeberria, 2014). Una explicación al respecto puede ser que este tipo de violencia está más aceptada socialmente y es menos castigada al ser difícil su verificación. Además, muchos agresores no son conscientes de que están ejerciendo violencia ya que ésta se asocia intrínsecamente a la presencia de daño físico (Martxueta y Etxeberria, 2014). Contrariamente, la violencia física acarrea consecuencias penales y legislativas severas, siendo más fácil su constatación y persecución (Anderson, 2014). A su vez, en la línea de otros estudios, esta victimización suele tener una duración limitada, sin reiteración ni prolongación en el tiempo (Marchueta, 2014). La violencia sufrida durante la adultez en forma de delitos de odio suele recibirse de forma puntual (Cereceda et al., 2018). Por su parte, la violencia experimentada durante la etapa escolar sí puede ser un poco más prolongada al tratarse de comportamientos perpetrados sin el conocimiento de las figuras de autoridad y no revelados por la víctima debido al miedo, la existencia de amenazas explícitas o la creencia de que no se contará con el apoyo de los maestros o los padres (Baruch-Domínguez, Infante-Xibille y Saloma-Zuñiga, 2016; Elipe et al., 2018; Rodríguez, 2018).

Los resultados obtenidos en función del género también revelan algunos aspectos que coinciden con la literatura científica. Independientemente del tipo de violencia, los hombres han sufrido mayores tasas de victimización exceptuando aquellos comportamientos relacionados con la exclusión o el aislamiento social, donde las mujeres presentan mayor prevalencia (Pichardo et al., 2007). Esta tendencia suele mantenerse en el tiempo, independientemente de si la victimización se ha producido durante la etapa escolar (Pace, D’Urso y Fontanesi, 2020) o durante la adultez (LoSchiavo, Halkitis y Kapadia, 2019). Así, los datos sobre delitos de odio proporcionados por el Ministerio del Interior indican que dos de cada tres delitos por orientación sexual son contra hombres (Cereceda et al., 2018). No obstante, estas cifras sólo tienen en consideración los actos denunciados y no los realmente acontecidos y puesto que las mujeres son más proclives a denunciar que los hombres esta diferencia de género todavía podía ser mayor. Una explicación a estas diferencias radica en que la violencia homofóbica esta intrínsecamente ligada a la trasgresión de los roles de género que cada cultura asocia a hombres y mujeres (Carrera-Fernández et al., 2018). En general, como el 
rol de género masculino es más rígido e inflexible, la probabilidad de que un hombre sea identificado como gay se incrementa sustancialmente (Weber y Gredig, 2018). La homofobia se fundamenta sobre la orientación sexual percibida por el agresor, por lo que algunos hombres heterosexuales que rompen con los roles de género también pueden sufrir este tipo de victimización (Poteat et al., 2014). Contrariamente, como el género femenino es más flexible muchas mujeres homosexuales pasan inadvertidas y sólo sufren violencia cuando verbalizan explícitamente su orientación sexual (Weber y Gredig, 2018).

En general, la victimización homofóbica sufrida por personas homosexuales y bisexuales tiene fuertes repercusiones sobre la salud mental. Así, las personas que han sufrido violencia verbal o física de forma severa tienen un nivel de autoestima significativamente menor que las persona no victimizadas o con una victimización puntual. Estos datos son coherentes con los trabajos de Huebner et al. (2004) y Marchueta (2014) quienes observan un descenso del nivel de autoestima en personas que han sufrido violencia a causa de su orientación sexual. También coinciden con los trabajos sobre acoso escolar que evidencian los efectos negativos de este tipo de violencia (DeLay, Hanish, Zhang y Martin, 2017). En contraposición, las personas que han recibido una victimización puntual y poco prolongada presentan niveles de autoestima que, si bien son ligeramente inferiores a las personas que no han recibido victimización, no difieren significativamente. Es probable que antes de ser victimizadas estas personas ya contaran con una buena autoestima que les proporcionase algunas herramientas personales para afrontar la situación, haciéndola pública y denunciando los hechos (Blais, Gervais y Hébert, 2014). Así, aunque la victimización repercute negativamente en la autoestima el nivel previo del que se parte hace esta disminución más sostenida.

Las personas que han sufrido violencia prolongada y severa presentan mayor sintomatología depresiva que las personas que no han recibido ninguna victimización, al igual que destaca el trabajo de Teasdale y Bradley-Engen (2010). Específicamente, estas personas presentan tristeza, auto-rechazo, sentimientos de culpa, aislamiento y mayor ideación suicida (Marchueta, 2014). Por su parte, las personas que han recibido una victimización leve sólo muestran síntomas depresivos si ha existido violencia física en forma de golpes, empujones o palizas. Es probable que algunas personas homosexuales y bisexuales crean que la victimización es algo intrínseco e inevitable al hecho de no ser heterosexuales, por lo que, desde el principio muchas personas se preparan y resignan a ser el blanco de las críticas y la violencia verbal (Baruch-Domínguez et al., 2016). Por el contrario, frente a la violencia física casi nadie está preparado y sus secuelas son mucho más difíciles de borrar.

Este estudio, como cualquier otro trabajo, posee algunas limitaciones. En primer lugar, aunque las conductas analizadas tienen un carácter retrospectivo que abarca todas las etapas del ciclo vital, no se tiene información sobre el momento exacto en el que se produjo. En futuros estudios sería conveniente precisar si esta victimización aconteció durante la etapa educativa, durante la adultez o en ambos periodos. A su vez, en lo que respecta al modo en que se perpetró, el estudio no evalúa si la violencia verbal ocurrió directamente o se produjo a través de las tecnologías de la información (redes sociales, aplicaciones de mensajería instantánea, etc.). Esta circunstancia tiene especial relevancia ya que, a diferencia de la victimización tradicional, la cibervictimización tiene algunas características específicas como 
la imposibilidad de escapar ante una violencia que puede darse en cualquier momento y prolongarse a lo largo de las 24 horas al día (Ortega-Barón, Buelga, Ayllón, Martínez-Ferrer y Cava, 2019). Finalmente, el carácter transversal del diseño impide el establecimiento de relaciones causa efecto, por lo que en futuros trabajos sería interesante realizar estudios longitudinales.

No obstante, y a pesar de estas limitaciones, este trabajo aporta nuevos datos sobre la prevalencia e influencia que ejerce la violencia homofóbica sobre la salud mental. Nuestro estudio confirma que la victimización homofóbica constituye una circunstancia experimentada por un elevado porcentaje de personas homosexuales y bisexuales; y se identifican los efectos negativos que esta tiene sobre la sintomatología depresiva y el nivel de autoestima. En general, las consecuencias psicopatológicas de esta violencia son relativamente estables independientemente de si ha acontecido en la etapa escolar o durante la adultez. Además, estas repercusiones negativas se incrementan en función de la gravedad y su prolongación temporal. Todo esto indica la necesidad de implementar programas educativos que prevengan la violencia o, al menos, reduzcan al máximo su prolongación en el tiempo. En la etapa escolar, contexto donde se producen los primeros actos violentos, sería necesario contar con profesores formados y sensibilizados que fuesen capaces de identificar y atajar los comportamientos violentos de forma temprana.

\section{Referencias}

Albaladejo-Blázquez, N., Ferrer-Cascales, R., Ruiz-Robledillo, N., Sánchez-SanSegundo, M., Fernández-Alcántara, M., Delvecchio, E. y Arango-Lasprilla, J. C. (2019). Health-related quality of life and mental health of adolescents involved in school bullying and homophobic verbal content bullying. International Journal of Environmental Research and Public Health, 16(14), 2622. doi:10.3390/ijerph16142622

Anderson, J. (2014). Providing a Safe Learning Environment for Queer Students in Canadian Schools: A Legal Analysis of Homophobic Bullying. Journal of LGBT Youth, 17(3), 212-243. doi: 10.1080/19361653.2013.879463

Ballester-Arnal, R., Morell-Mengual, V., Gil-Llario, M. D., Salmerón-Sánchez, P. y Castro-Calvo, J. (2015). Propiedades psicométricas preliminares de una escala de victimización homosexual. Póster presentado a la XXIV Reunión de la SPCV, Segorbe, España.

Baruch, R. (2012). La Encuesta nacional sobre bullying homofóbico. Recuperado de http://www.adilmexico.com/ wp-content/uploads/2015/08/Bullying-homofobico-114-May-12.pdf

Baruch-Domínguez, R., Infante-Xibille, C. y Saloma-Zuñiga, C. E. (2016). Homophobic bullying in Mexico: Results of a national survey. Journal of LGBT Youth, 13(1-2), 18-27. doi: 10.1080/19361653.2015.1099498

Blais, M., Gervais, J. y Hébert, M. (2014). Internalized homophobia as a partial mediator between homophobic bullying and self-esteem among youths of sexual minorities in Quebec (Canada). Ciência \& Saúde Coletiva, 19(3), 727-735. doi: 10.1590/1413-81232014193.16082013

Blondeel, K., de Vasconcelos, S., García-Moreno, C., Stephenson, R., Temmermana, M., y Toskinb, I. (2018). Violence motivated by perception of sexual orientation and gender identity: a systematic review. Bulletin of the World Health Organization, 96, 29-41. doi: 10.2471/BLT.17.197251

Carrera-Fernández, M. V., Cid-Fernández, X. M., Almeida, A., González-Fernández, A. y Lameiras-Fernández, M. (2018). Attitudes Toward Cultural Diversity in Spanish and Portuguese Adolescents of Secondary Education: The Influence of Heteronormativity and Moral Disengagement in School Bullying. Revista de Psicodidáctica, 23(1), 17-25. doi: 10.1016/j.psicoe.2017.07.002 
Cereceda, J., Sánchez, F., Herrera, D., Morán, C., Fernández, T., Martínez, F. (...) Gómez, M. A. (2018). Informe sobre la evolución de los delitos de odio en España. Recuperado de http://www.interior.gob.es/documents/642012/3479677/informe+2018/ab86b6d9-090b-465b-bd14-cfcafccdfebc

D'haese, L., Dewaele, A. y Van Houtte, M. (2016). Homophobic Violence, Coping Styles, Visibility Management, and Mental Health: A Survey of Flemish Lesbian, Gay, and Bisexual Individuals. Journal of Homosexuality, 63(9), 1211-1235. doi: 10.1080/00918369.2016.1150057.

DeLay, D., Hanish, L.D., Zhang, L. y Martin, C. L. (2017). Assessing the Impact of Homophobic Name Calling on Early Adolescent Mental Health: A Longitudinal Social Network Analysis of Competing Peer Influence Effects. Journal of Youth and Adolescence, 46, 955-969. Doi: 10.1007/s10964-016-0598-8

Elipe, P., de la Oliva, M. y Del Rey, R. (2018). Homophobic bullying and cyberbullying: Study of a silenced problem. Journal of homosexuality, 65(5), 672-686. doi:10.1080/00918369.2017.1333809

FELCTB. (2012). Acoso escolar homofóbico y riesgo de suicidio en adolescentes y jóvenes LGB. Recuperado de http://www.felgtb.org/rs/1584/d112d6ad-54ec-438b-9358-4483f9e98868/91c/filename

Garaigordobil, M. y Larrain, E. (2020). Bullying and cyberbullying in LGBT adolescents: Prevalence and effects on mental health. Comunicar, 28(62), 77-87. doi: 10.3916/C62-2020-07

Herrero, J. y Gracia, E. (2007). Una medida breve de la sintomatología depresiva (CESD-7). Salud Mental, 30(5), 40-46.

Huebner, D. M., Rebchook, G.y Kegeles, S. (2004). Experiences of harassment, discrimination, and physical violence among young gay and bisexual men. American Journal of Public Health, 94, 1200-1203. doi:10.2105/ AJPH.94.7.1200

Kosciw, J. G., Greytak, E. A., Giga, N. M., Villenas, C. y Danischewski, D. J. (2016). The 2015 national school climate survey: The experiences of lesbian, gay, bisexual, transgender, and queer youth in our nation's schools. New York, NY: GLSEN. Recuperado de https://www.glsen.org/article/2015-national-school-climate-survey

Lehavot, K. y Simoni, J. M. (2011). The impact of minority stress on mental health and substance use among sexual minority women. Journal of Consulting and Clinical Psychology, 79(2), 159-170. doi:10.1037/a0022839

LoSchiavo, C., Halkitis, P. N. y Kapadia, F. (2019). Sexual orientation and gender identity victimization among young adults in the New York City metropolitan area: The P18 cohort study. Psychology of Sexual Orientation and Gender Diversity, 6(4), 399-407. doi: 10.1037/sgd0000336

Marchueta, A. (2014). Consecuencias del bullying homofóbico retrospectivo y los factores psicosociales en el bienestar psicológico de sujetos LGB. Revista de Investigación Educativa, 32(1), 255-271. doi: 10.6018/ rie.32.1.168461

Martín-Albo, J., Núñez, J. L., Navarro, J. G. y Grijalvo, F. (2007). The rosenberg self-esteem scale: Translation and validation in university students. The Spanish Journal of Psychology, 10(2), 458-467. doi: 10.1017/ S1138741600006727

Martxueta, A. y Etxeberria, J. (2014). Análisis diferencial retrospectivo de las variables de salud mental en lesbianas, gais y bisexuales (LCB) víctimas de bullying homofóbico en la escuela. Revista de Psicopatología y Psicología Clínica, 19(1), 23-35. doi: 10.5944/rppc.vol.19.num.1.2014.12980

Ortega-Barón, J., Buelga, S., Ayllón, E., Martínez-Ferrer, B. y Cava, M. J. (2019). Effects of intervention program Prev@cib on traditional bullying and cyberbullying. International Journal of Environmental Research and Public Health, 16(4), 527. doi: 10.3390/ijerph76040527

Pace, U., D'Urso, G. y Fontanesi, L. (2020). The vicissitudes of homophobic victimization in adolescence: an explorative study. Frontiers in psychology, 17, 43. doi: 10.3389/fpsyg.2020.00043

Petrou, P., \& Lemke, R. (2018). Victimisation and life satisfaction of gay and bisexual individuals in 44 European countries: the moderating role of country-level and personlevel attitudes towards homosexuality. Culture, Health and Sexuality, 20(6), 640-657. doi: 10.1080/13691058.2017.1368710

Pichardo, J. I., de Stéfano, M., Faure, J., Sáenz, M. y Williams, J. (2015). Abrazar la diversidad: Propuestas para una educación libre de acoso homofóbico y transfóbico. Madrid: Instituto de la mujer y para la igualdad de oportunidades. 
Pichardo, J.l., Molinuelo, B., Rodríguez, P.O., Martín, N. y Romero, M. (2007). Actitudes ante la diversidad sexual de la población adolescente de Coslada (Madrid) y San Bartolomé de Tirajana (Gran Canaria). Madrid: FELGTB.

Platero, R. (2008). La homofobia como elemento clave del acoso escolar homofóbico. Algunas voces desde Rivas Vaciamadrid. Información Psicológica, 94, 71-83.

Poteat, V. P., Scheer, J. R., DiGiovanni, C. D. y Mereish, E. H. (2014). Short-Term Prospective Effects of Homophobic Victimization on the Mental Health of Heterosexual Adolescents. Journal of Youth and Adolescence, 43, 1240-1251 doi: 10.1007/s10964-013-0078-3

Radloff, L. S. (1977). The CES-D scale: A self-report depression scale for research in the general population. Applied Psychological Measurement, 7(3), 385-401. doi: 10.1177/014662167700100306

Rodríguez, L. M. (2018). Bullying homofóbico en México a nivel de secundaria: el contexto de Nuevo León. Revista de Psicología, 36(2), 631-659. doi: 10.18800/psico.201802.009

Rosenberg, M. (1965). Society and the adolescent self-image. Princeton, N.J.: Princeton University Press.

Teasdale, B. y Bradley-Engen, M. (2010). Adolescent same-sex attraction and men- tal health: The role of stress and support. Journal of Homosexuality, 57(2), 287-309. doi: 10.1080/00918360903489127

UNESCO. (2015). La violencia homofóbica y transfóbica en el ámbito escolar: hacia centros educativos inclusivos y seguros en América Latina. París: UNESCO.

Weber, P. y Gredig, D. (2018). Prevalence and predictors of homophobic behavior among high school students in Switzerland. Journal of Gay \& Lesbian Social Services, 30(2), 128-153. doi: 10.1080/10538720.2018.1440683

Worthen, M.C.F., Lingiardi, V. y Caristo, C. (2017). The Roles of Politics, Feminism, and Religion in Attitudes Toward LCBT Individuals: A Cross-Cultural Study of College Students in the USA, Italy, and Spain. Sexuality Research and Social Policy, 14, 241-258. doi. 10.1007/s13178-016-0244-y 